\title{
Multilayer Codes for Synchronization from Deletions
}

\author{
Mahed Abroshan \\ University of Cambridge \\ ma675@cam.ac.uk
}

\author{
Ramji Venkataramanan \\ University of Cambridge \\ ramji.v@eng.cam.ac.uk
}

\author{
Albert Guillén i Fàbregas \\ ICREA \& Universitat Pompeu Fabra \\ University of Cambridge \\ guillendieee.org
}

\begin{abstract}
A coding scheme is proposed for synchronization from a small number of deletions via a one-way error-free link. The scheme is based on multiple layers of Varshamov-Tenengolts codes combined with off-the-shelf linear error-correcting codes.
\end{abstract}

\section{INTRODUCTION}

Consider two remote nodes having binary sequences $X$ and $Y$, respectively, where $Y$ is an edited version of $X$. In this paper, we consider the edits to be deletions. Let the length of $X$ be $n$ bits, and the number of deletions be $k$. Thus, $Y$ is a sequence of length $m=(n-k)$, obtained by deleting $k$ bits from $X$. In the synchronization model shown in Fig. 1 , the node with $X$ (the "encoder") sends a message $M$ via an error-free link to the other node (the "decoder"), which attempts to reconstruct $X$ using $M$ and $Y$. The goal is to design a scheme so that the decoder can reconstruct $X$ with minimal communication, i.e., we want to minimize the number of bits used to represent the message $M$.

The deletion model considered here is a simplified version of the general file synchronization problem where the edits can be a combination of deletions, insertions, and substitutions. The general synchronization problem has a number of applications including file backup (e.g., Dropbox) and file sharing. Various forms of the synchronization model have been studied in previous works; see, e.g., [1]-[4]. A number of these works allow two-way interaction between the encoder and decoder.

In contrast, we seek codes for one-way synchronization: the message $M$ is produced by the encoder using only $X$, with no knowledge of $Y$ except its length $m$. We assume that the decoder knows $n$, so it can infer the number of deletions $k=(n-m)$. The message $M$ belongs to a finite set $\mathcal{M}$ with cardinality $|\mathcal{M}|$. The synchronization rate is defined as $R=\frac{\log _{2}|\mathcal{M}|}{n}$. We would like to design a code for reliable synchronization with $R$ as small as possible; $R=1$ is equivalent to the encoder sending the entire string $X$.

In this paper, we construct a code for synchronization from deletions when the number of deletions $k$ is small compared to $n$. The output of the decoder is a small list of sequences that is guaranteed to contain the correct sequence $X$. Though we do not provide theoretical bounds on the list size, we observe from simulations that with a careful choice of code parameters, the list size rarely exceeds 2 or 3 ; for reasonably large $n$, the list size can be made 1, i.e., $X$ is exactly reconstructed. For

This work has been funded in part by the European Research Council under ERC grant agreement 259663 and by the Spanish Ministry of Economy and Competitiveness under grant TEC2016-78434-C3-1-R.

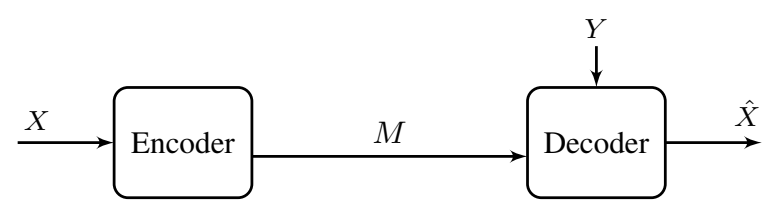

Fig. 1: Synchronization Model

example, we construct a code of length $n=378$ that can synchronize from $k=7$ deletions with $R=0.365$, and a length $n=2800$ code which can synchronize from $k=10$ deletions with $R=0.135$. (Details in Section IV.)

Overview of code construction: The starting point for our code construction is the family of Varshamov-Tenengolts (VT) codes [5], [6]. Each VT code is a single deletion correcting code. As observed in [7], the VT family gives an elegant way to exactly synchronize from a single deletion: the encoder simply sends the VT syndrome of the sequence $X$. The VT syndrome, defined in the next section, indicates which VT code $X$ belongs to. The decoder then uses the single deletion correcting property of the VT code to recover the deleted bit.

In our model, the code needs to synchronize from $k>1$ deletions. The encoder sends the VT syndromes of various substrings of $X$ to the decoder. The length $n$ sequence $X$ is divided into smaller chunks of $n_{c}$ bits each. The encoder then computes VT syndromes for two kinds of substrings: blocks which are composed of adjacent chunks, and chunk-strings which are composed of well-separated chunks. Fig. 2 shows an example where $X$ of length 12 is divided into 4 length-3 chunks. The blocks $B_{1}$ and $B_{2}$ are each formed by combining two adjacent chunks, while the chunk-strings $C_{1}$ and $C_{2}$ are each formed by combining two alternate chunks. In this case, the encoder sends the VT syndromes of $B_{1}, B_{2}, C_{1}$, and $C_{2}$.

The intersecting VT constraints of the blocks and the chunk-strings help the decoder to iteratively determine the approximate locations of the edits. The VT syndromes serve a dual purpose: i) they can be used to recover deleted bits in blocks or chunk-strings inferred to have a single deletion; this recovery may result in new blocks and chunk-strings with a single deletion; ii) the VT syndromes also act as checks that eliminate a large number of deletion patterns, allowing the decoder to localize the deletions to a relatively small set of chunks. The final ingredient of the message is a parity check syndrome of $X$ using a linear code. This is used to recover the deletions in chunks that still remain uncertain at the decoder after processing the intersecting VT constraints. 


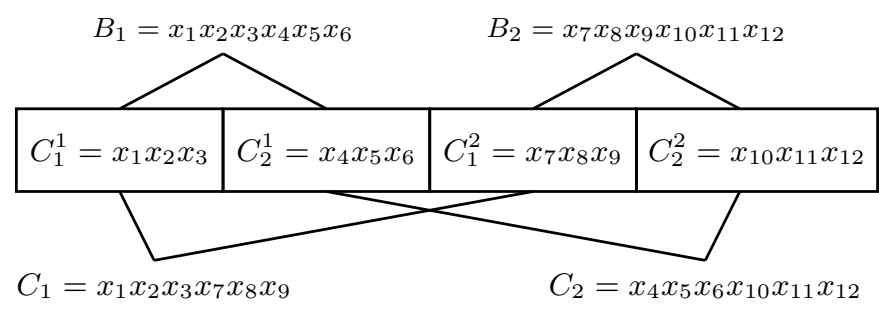

Fig. 2: Blocks and chunk-strings structure for the example where $l_{1}=l_{2}=2$

We refer to this code construction as a two-layer code as the chunks are combined to form two kinds of intersecting substrings. The construction can be generalized to combine chunks in multiple ways to form many layers of intersecting substrings. Increasing the number of constraints in the code improves its synchronization capability at the cost of increasing the rate and decoding complexity.

The problem of one-way synchronization from $k$ deletions is closely related to the problem of communicating over a deletion channel that deletes $k$ bits from a length $n$ codeword [8]. The channel coding version of the proposed code construction will be discussed in an extended version of this paper.

Notation: We denote scalars using lower-case letters and sequences using capital letters. The subsequence of $X$ from index $i$ to index $j$ is denoted by $X(i: j)=x_{i} x_{i+1} \cdots x_{j}$. Matrices are denoted by bold capitals. We use brackets for merging sequences, so $X=\left[X_{1}, \cdots, X_{u}\right]$ is a super-sequence defined by concatenating the sequences $X_{1}, \cdots, X_{u}$.

\section{Code Construction And Encoding}

We begin with a review of VT codes. The VT syndrome of a binary sequence $W=\left(w_{1}, \ldots, w_{n}\right)$ is defined as

$$
\operatorname{syn}(W)=\sum_{j=1}^{n} j w_{j} \quad(\bmod (n+1)) .
$$

For positive integers $n$ and $0 \leq s \leq n$, we define the VT code of length $n$ and syndrome $s$, denoted by

$$
\mathcal{V} \mathcal{T}_{s}(n)=\left\{W \in\{0,1\}^{n}: \operatorname{syn}(W)=s\right\},
$$

as the set of sequences $W$ of length $n$ for which $\operatorname{syn}(W)=s$.

The $n+1$ sets $\mathcal{V} \mathcal{T}_{s}(n) \subset\{0,1\}^{n}$, for $0 \leq s \leq n$, partition the set of all sequences of length $n$. Each of these sets $\mathcal{V} \mathcal{T}_{s}(n)$ is a single-deletion correcting code. The complexity of the VT decoding algorithm is linear in the code length $n$ [9].

Constructing the message: The message $M$ generated by the encoder consists of three parts, denoted by $M_{1}, M_{2}$, and $M_{3}$. The first part comprises the VT syndromes of the blocks, the second part comprises the VT syndromes of the chunkstrings, and the third part is the parity check syndrome of $X$ with respect to a linear code.

The first step is to divide $X=x_{1} x_{2} \cdots x_{n}$ into $l_{1}$ equalsized blocks (assume that $n$ is divisible by $l_{1}$ ). The length of each block is denoted by $n_{b}=\frac{n}{l_{1}}$. For $1 \leq i \leq l_{1}$, the $i$ th block is denoted by $B_{i}=X\left((i-1) n_{b}+1: i n_{b}\right)$, and its VT syndrome is $s_{B_{i}}=\operatorname{syn}\left(B_{i}\right)$. The first part of the message is the collection of VT syndromes for the $l_{1}$ blocks, i.e., $M_{1}=$ $\left\{s_{B_{1}}, s_{B_{2}}, \cdots, s_{B_{l_{1}}}\right\}$. Since each $s_{B_{i}}$ is an integer between 0 and $n_{b}$, the number of bits required to represent the VT syndromes of the $l_{1}$ blocks is $l_{1}\left\lceil\log \left(n_{b}+1\right)\right\rceil$.

For the second part of the message, we divide each block into $l_{2}$ chunks, each of size $n_{c}$ bits. We assume that $\frac{n}{l_{1}}$ is divisible by $l_{2}$; the length of $X$ is $n=n_{c} l_{1} l_{2}$. For $1 \leq j \leq l_{2}$, the $j$ th chunk within the $i$ th block is denoted by

$$
C_{j}^{i}=X\left((i-1) n_{b}+(j-1) n_{c}+1:(i-1) n_{b}+j n_{c}\right) .
$$

The $j$ th chunk-string is then formed by concatenating the $j$ th chunk from each of the $l_{1}$ blocks. That is, the $j$ th chunk string $C_{j}=\left[C_{j}^{1}, C_{j}^{2}, \cdots, C_{j}^{l_{1}}\right]$, for $1 \leq j \leq l_{2}$. Fig. 2 shows the blocks and the chunk-strings in an example where $X$ of length $n=12$ is divided into $l_{1}=2$ blocks, each of which is divided into $l_{2}=2$ chunks of $n_{c}=3$ bits.

The second part of the message is the collection of VT syndromes for the $l_{2}$ chunk-strings, i.e., $M_{2}=$ $\left\{s_{C_{1}}, s_{C_{2}}, \cdots, s_{C_{l_{2}}}\right\}$, where $s_{C_{j}}$ denotes the VT syndrome of the $j$ th chunk string. Since the length of each chunk-string is $n_{c} l_{1}$, each $s_{C_{j}}$ is an integer between 0 and $n_{c} l_{1}$. Therefore the number of bits required to represent the VT syndromes of the $l_{2}$ chunk-strings is is $l_{2}\left\lceil\log \left(n_{c} l_{1}+1\right)\right\rceil$.

The final part of the message is the parity check syndrome of $X$ with respect to a linear code. Consider a linear code of length $n$ with parity check matrix $\boldsymbol{H} \in\{0,1\}^{z \times n}$. Then $M_{3}=\boldsymbol{H} X$ is the third component of $M$. The coset of the linear code containing $X$ will be used as an erasure correcting code. In our experiments in Sec. IV, the linear code is chosen to be either a Reed-Solomon code, or a random linear code defined by a random binary parity check matrix. The number of bits in $M_{3}$ is equal to the number of rows of $\boldsymbol{H}$, i.e., number of binary parity checks in the code, $z$. The overall number of bits required to represent the message $M=\left[M_{1}, M_{2}, M_{3}\right]$ is

$$
l_{1}\left\lceil\log _{2}\left(n_{b}+1\right)\right\rceil+l_{2}\left\lceil\log _{2}\left(n_{c} l_{1}+1\right)\right\rceil+z .
$$

Since $n_{b}=n_{c} l_{2}$, normalizing by $n=n_{c} l_{1} l_{2}$ gives the synchronization rate $R$ of our scheme

$$
R=\frac{z}{n}+\frac{\left\lceil\log _{2}\left(n_{c} l_{2}+1\right)\right\rceil}{n_{c} l_{2}}+\frac{\left\lceil\log _{2}\left(n_{c} l_{1}+1\right)\right\rceil}{n_{c} l_{1}} .
$$

Example 1. Suppose that we want to design a code for synchronizing a sequence of length $n=60$ from $k=4$ deletions. Choose the chunk length $n_{c}=4$, so that there are 15 chunks in the string. Divide the string into $l_{1}=5$ blocks, each comprising $l_{2}=3$ chunks. Thus there are 5 blocks each consisting of 3 adjacent chunks, and 3 chunk-strings each consisting of 5 separated chunks.

We use a Reed-Solomon code defined over $G F\left(2^{4}\right)$ with length $2^{4}-1=15$. We also choose the parity check matrix to have 4 parity check equations in $G F\left(2^{4}\right)$, so we can recover 4 erased chunks using this Reed-Solomon code.

Assume that the sequence $X$ in $G F\left(2^{4}\right)$ is

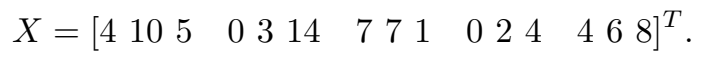




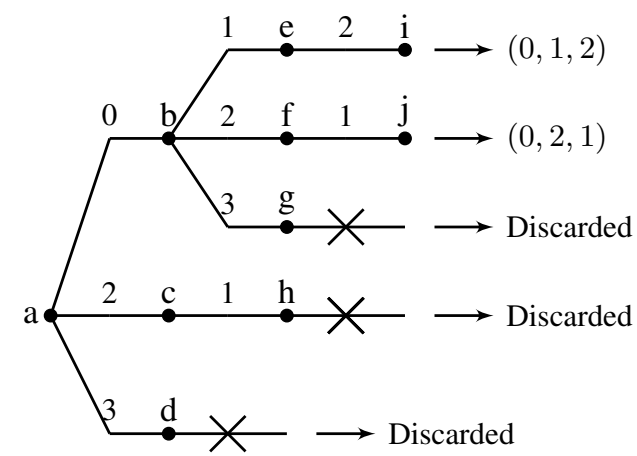

Fig. 3: Tree representing the valid block vectors for Example 2.

Each symbol above represents a chunk of $n_{c}=4$ bits. The first block [4 10 5] in binary is $B_{1}=01001010$ 0101. The $V T$ syndrome of this sequence is $s_{B_{1}}=\operatorname{syn}\left(B_{1}\right)=10$. The $V T$ syndromes of the other four blocks are 6,3,4, and 11, respectively. We therefore have $M_{1}=\{10,6,3,4,11\}$.

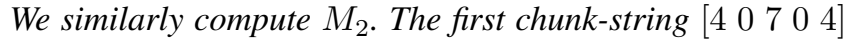
in binary is $C_{1}=0100000001110000$ 0100, with VT syndrome $s_{C_{1}}=11$. Computing the VT syndromes of the other chunk-strings in a similar manner, we get $M_{2}=\{11,20,4\}$.

The final part of the message is the syndrome of $X$ with respect to the Reed-Solomon parity check matrix. We use the following parity check matrix $\boldsymbol{H}$ in $G F\left(2^{4}\right)$ :

$$
\boldsymbol{H}=\left[\begin{array}{cccccc}
1 & 1 & 1 & 1 & \ldots & 1 \\
1 & 2 & 4 & 8 & \ldots & 2^{14} \\
1 & 4 & 3 & 12 & \ldots & 2^{2(14)} \\
1 & 8 & 12 & 10 & \cdots & 2^{3(14)}
\end{array}\right]
$$

to compute $M_{3}=\boldsymbol{H} X=[11,6,13,2]^{T}$. As $z=16$ bits are needed to represent the parity check syndrome, the total number of bits to convey the message is $5\lceil\log (13)\rceil+$ $3\lceil\log (21)\rceil+16=51$ bits.

\section{DECODING ALGORITHM}

The goal of the decoder is to recover $X$ given $Y, n$ and the message $M=\left[M_{1}, M_{2}, M_{3}\right]$. From $M_{1}, M_{2}$, the decoder knows the VT syndrome of each block and each chunk-string. Using this, the decoder first finds all possible configurations of deletions across blocks, and then for each of these configurations, it finds all possible chunk deletion patterns. Since each chunk is the intersection of a block and a chunk-string, each chunk plays a role in determining exactly two VT syndromes. The intersecting construction of blocks and chunk-strings enables the decoder to iteratively recover the deletions in a large number of cases. The decoder is then able to localize the positions of the remaining deletions to within a few chunks. These chunks are considered erased, and are finally recovered by the erasure-correcting code. The decoding algorithm consists of six steps, as described below.

\section{Step 1: Block boundaries}

In the first step, the decoder produces a list of candidate block-deletion patterns $V=\left(a_{1}, \cdots, a_{l_{1}}\right)$ compatible with
$Y$, where $a_{i}$ is the number of deletions in the $i$ th block. Each pattern in the list should satisfy $\sum_{i=1}^{l_{1}} a_{i}=k$ with $0 \leq a_{i} \leq k$. The list of candidates always includes the true block-deletion pattern. It is convenient to represent the candidate blockdeletion patterns as branches on a tree of depth $l_{1}$, as shown in Fig. 3. At every level (block) $i=1, \ldots, l_{1}$, branches are added and labeled with all possible values of $a_{i}$. Specifically, the tree is constructed as follows.

Depth 1 of the tree: Consider the first $n_{b}$ received bits $Y\left(1: n_{b}\right)$, compute its VT syndrome $u=\operatorname{syn}\left(Y\left(1: n_{b}\right)\right)$ and compare it with $s_{B_{1}}$, the correct syndrome of the first block. There are two alternatives for the $k$ branches of the first level.

1) $u=s_{B_{1}}$ : First, the decoder adds a branch with $a_{1}=0$, corresponding to the case that the first $n_{b}$ bits are deletion-free. The first block cannot have just one deletion, because in this case the single-deletion correcting property of the VT code would imply that $u \neq s_{B_{1}}$. However, it is possible that two or more than two deletions happened in block one, and by considering additional bits from the next block, the VT-syndrome of first $n_{b}$ bits accidentally matches with $s_{B_{1}}$. For example, consider blocks of length $n_{b}=4$, and let the first two blocks of $X$ be $01001111 \ldots$, with the underlined bits deleted we get $Y=001111 \ldots$. In this case $u=s_{B_{1}}=2$. The decoder thus adds a branch for $a_{1}=0,2, \ldots, k$.

2) $u \neq s_{B_{1}}$ : Block one contains one or more deletions and the decoder adds a branch for $a_{1}=1,2, \ldots, k$.

Depth $i+1,1 \leq i<l_{1}$ : Assume that we have constructed the tree up to depth $i$. Consider a branch of the tree at depth $i$ with the number of deletions in blocks 1 through $i$ given by $a_{1}, a_{2}, \cdots, a_{i}$, respectively. This gives us the starting position of block $(i+1)$ in $Y$. Denote this starting position by

$$
p_{i+1}=n_{b} i-d_{i}+1 \text {. }
$$

where $d_{i}=\sum_{j=1}^{i} a_{j}$ is the number of deletions on the branch up to block $i$. Compute the VT syndrome of next $n_{b}$ bits $u=$ syn $\left(Y\left(p_{i+1}: p_{i+1}+n_{b}-1\right)\right)$. There are two alternatives:

1) $u=s_{B_{i+1}}$ : If $\left(k-d_{i}\right)<2$ then the only possibility is that $\overline{a_{i+1}}=0$. Instead, if $\left(k-d_{i}\right) \geq 2, k-d_{i}-1$ branches are added for $a_{i+1}=0,2, \ldots, k-d_{i}$.

2) $u \neq s_{B_{i+1}}$ : If $\left(k-d_{i}\right)>0$ then there are $\left(k-d_{i}\right)$ possibilities at this branch: the $i$ th block can have $1,2, \cdots,\left(k-d_{i}\right)$ deletions. If $\left(k-d_{i}\right)=0$, it is assumed this is an invalid branch, and the path is discarded.

Example 2. Assume $k=3$ deletions, $l_{1}=3$ blocks, and that the true deletion pattern is $(0,2,1)$, i.e., there are zero deletions in the first block, two deletions in second block, and one deletion in third block. The tree constructed by the decoder depends on the underlying sequences $X$ and $Y$. In Fig. 3, we illustrate one possible tree constructed for this scenario without explicitly specifying $X$ and $Y$.

Assume that in the first step, the syndrome matches with $s_{B_{1}}$, so we have $a_{1}=0,2$, or 3 . At node $b$ (corresponding to $a_{1}=0$ ), suppose that the syndrome does not match with $s_{B_{2}}$, so we have $a_{2}=1,2$, or 3 . Now suppose that at nodes 
$c$ and $d$, the syndrome does not match with $s_{B_{2}}$. At node $d$, $a_{1}=3$, so there are no more deletions available for the second block; so this branch is discarded. At node c, $a_{1}=2$, so the only possibility is one deletion in the second block. Then if the syndrome at node $h$ does not match $s_{B_{3}}$, the branch is discarded. At nodes $e$ and $f$, we assign the remaining deletions to the last block. At node g, the syndrome does not match with $a_{3}$, and the branch is discarded.

\section{Step 2: Primary fixing of blocks}

Denote by $r_{1}$ the size of the list after the first step and denote the corresponding block-deletion patterns by $V_{1}, \cdots, V_{r_{1}}$. In this second step, for each of the block-deletion patterns, we restore the deleted bit in blocks containing a single deletion by using the VT decoder. Specifically, for every block-deletion pattern $V=\left(a_{1}, \cdots, a_{l_{1}}\right)$, let the $i$ th block of $Y$ with respect to $V$ be $S=Y\left(p_{i}: p_{i}+n_{b}-a_{i}-1\right)$ where $p_{i}$ is the starting position of the $i$ th block in $Y$, defined analogously to (8). If $a_{i}=1$, feed the sequence $S$ to the VT decoder and in $Y$, replace $S$ with the decoded sequence. After this, the $i$ th block in $Y$ is deletion free, so update the block-deletion pattern $V$ by setting $a_{i}=0$. We carry out this procedure for all blocks with one deletion in $V$. This results in a sequence $\hat{Y}$, which is obtained from $Y$ by recovering the single-deletion blocks corresponding to block-deletion pattern $V$. Denote the updated version of block-deletion pattern $V$ by $\hat{V}$. Thus at the end of this step, we have $r_{1}$ updated candidate sequences $\hat{Y}_{1}, \cdots, \hat{Y}_{r_{1}}$ with corresponding block-deletion patterns $\hat{V}_{1}, \cdots, \hat{V}_{r_{1}}$.

Example 3. Consider the code of Example 1 with $l_{1}=5$ blocks, and $k=4$ deleted bits. If the list of block-deletion patterns at the end of the first step is $V_{1}=(1,1,1,1,0), V_{2}=$ $(1,1,2,0,0), V_{3}=(1,2,1,0,0), V_{4}=(2,0,2,0,0)$, then the updated list of block-deletion patterns is $\hat{V}_{1}=(0,0,0,0,0)$, $\hat{V}_{2}=(0,0,2,0,0), \hat{V}_{3}=(2,0,0,0,0), \hat{V}_{4}=(2,0,2,0,0)$.

\section{Step 3: Chunk Boundaries}

In this step, for each updated block-deletion pattern $\hat{V}$ and the corresponding $\hat{Y}$, we list all possible allocations of deletions across chunks. More precisely, for each pair $(\hat{Y}, \hat{V})$ we list all possible $l_{1} \times l_{2}$ matrices $\boldsymbol{A}=\left(a_{i j}\right)$, where $a_{i j}$ is the number of deletions in the $j$ th chunk of the $i$ th block, such that $\sum_{j=1}^{l_{2}} a_{i j}=a_{i}$, the $i$ th entry of $\hat{V}$. The $j$ th column of matrix $\boldsymbol{A}$, specifies the number of deletions in the $l_{1}$ chunks of the $j$ th chunk-string. For example, some of the possible matrices for $\hat{V}_{4}=(2,0,2,0,0)$ in Example 3 are

$$
\boldsymbol{A}_{1}=\left[\begin{array}{lll}
1 & 1 & 0 \\
0 & 0 & 0 \\
0 & 1 & 1 \\
0 & 0 & 0 \\
0 & 0 & 0
\end{array}\right] \quad \boldsymbol{A}_{2}=\left[\begin{array}{lll}
2 & 0 & 0 \\
0 & 0 & 0 \\
0 & 1 & 1 \\
0 & 0 & 0 \\
0 & 0 & 0
\end{array}\right] \quad \boldsymbol{A}_{3}=\left[\begin{array}{lll}
1 & 0 & 1 \\
0 & 0 & 0 \\
1 & 0 & 1 \\
0 & 0 & 0 \\
0 & 0 & 0
\end{array}\right] .
$$

The algorithm that lists all chunk-deletion matrices $\boldsymbol{A}$ compatible with a given block-deletion pattern $\hat{V}=\left(a_{1}, \ldots, a_{l_{1}}\right)$ is completely analogous to the tree construction described in Step 1 . In this case, for each block-deletion pattern $\hat{V}$, another tree will be constructed, with each path in the tree representing a valid chunk-deletion matrix $\boldsymbol{A}$. A detailed description of this tree construction is given in $[10, \mathrm{p} .4]$

\section{Step 4: Iterative correction of blocks and chunk-strings}

At the end of step 3, the decoder provides a list of pairs $(\hat{Y}, \boldsymbol{A})$, where $\hat{Y}$ is a candidate sequence to be decoded using the chunk-deletion pattern matrix $\boldsymbol{A}$, with $a_{i j}$ being the number of deletions in the $j$ th chunk of the $i$ th block. Denote the number of such pairs in the list by $r_{3}$.

Similarly to step 2, in step 4 we use the VT syndromes (known from $M_{1}$ and $M_{2}$ ) to recover deletions in blocks and chunk-strings for which the matrix $\boldsymbol{A}$ indicates a single deletion. Whenever a deletion recovered using a VT decoder lies in a chunk different from the one indicated by $\boldsymbol{A}$, the candidate is discarded. Simulations indicate that this is an effective way of discarding several invalid candidates. The iterative procedure is described in detail in [10, p.5]. Denote the updated candidate pairs at the end of this procedure by $(\tilde{Y}, \tilde{\boldsymbol{A}})$, and assume there are $r_{4}$ of them.

As an illustrative example, consider the three chunkmatrices given in (9). In $\boldsymbol{A}_{1}$, we can successfully recover all the deletions. In $\boldsymbol{A}_{2}$, we can only fix two deletions in the third block. However, for $\boldsymbol{A}_{3}$, we cannot recover any of the deletions. Thus, the updated $\tilde{\boldsymbol{A}}$ matrices are

$$
\tilde{\boldsymbol{A}}_{1}=\left[\begin{array}{lll}
0 & 0 & 0 \\
0 & 0 & 0 \\
0 & 0 & 0 \\
0 & 0 & 0 \\
0 & 0 & 0
\end{array}\right] \quad \tilde{\boldsymbol{A}}_{2}=\left[\begin{array}{lll}
2 & 0 & 0 \\
0 & 0 & 0 \\
0 & 0 & 0 \\
0 & 0 & 0 \\
0 & 0 & 0
\end{array}\right] \quad \tilde{\boldsymbol{A}}_{3}=\left[\begin{array}{lll}
1 & 0 & 1 \\
0 & 0 & 0 \\
1 & 0 & 1 \\
0 & 0 & 0 \\
0 & 0 & 0
\end{array}\right] \text {. }
$$

\section{Step 5: Replacing deletions with erasures}

In this step, for each of the $r_{4}$ surviving pairs $(\tilde{Y}, \tilde{\boldsymbol{A}})$, we replace each chunk of $\tilde{Y}$ that still contains deletions with $n_{c}$ erasures. Hence, if there are $\nu$ chunks with deletions (where $0 \leq \nu \leq k$ ), the resulting sequence will have length $n$, with $n_{c} \nu$ erasures and no deletions. Notice that this operation of replacing with erasures can be performed without ambiguity since $\tilde{\boldsymbol{A}}$ precisely indicates the starting position of each chunk and also the number of deletions within that chunk.

The purpose of the linear code is to recover from the remaining erasures. The minimum distance of the linear code should be large enough to guarantee that we can resolve all the $\nu n_{c}$ erased bits. In Example 1 with four deletions, we will have at most $\nu=4$ erased chunks, so we choose a Reed-Solomon code with 4 parity check equations in $G F\left(2^{4}\right)$. Some invalid candidates may be discarded in the process of correcting the erasures as we may find that the parity check equations cannot be solved.

\section{Step 6: Discarding invalid candidates}

The reconstructed sequences at the end of Step 5, denoted by $\hat{X}$, all have length $n$ and are deletion free. For each of the $r_{5}$ sequences $\hat{X}$, we check the VT and parity-check constraints for each of the block and chunk-strings and discard those 
not meeting any of the constraints. The surviving $r_{6}$ distinct sequences comprise the final list produced by the decoder.

The final list of reconstructed sequences comprises all length- $n$ sequences that can be obtained by adding $k$ bits to $Y$ and also satisfy all the VT and parity check constraints. The correct sequence is always among the $r_{6}$ candidates. The synchronization algorithm is said to be zero-error if and only if $r_{6}=1$ for all sequences and deletion patterns. When $r_{6}>1$, the list size can be further reduced if additional hash functions or cyclic redundancy checks are available from the encoder.

\section{NUMERICAL EXAMPLES}

To understand the effect of the various system parameters, we considered the setups shown in Table I. For each setup, the performance was recorded over $10^{6}$ simulation trials. In each trial, the sequence $X$ and the $k$ deletion locations were chosen independently and uniformly at random. For the first five setups, we used parity check constraints from a ReedSolomon code over $G F\left(2^{n_{c}}\right)$ with code length $\left(2^{n_{c}}-1\right)$. For example, setup 5 uses 7 parity check constraints from a ReedSolomon code over $G F\left(2^{6}\right)$, corresponding to $z=42$ parity bits. In the last two setups, denoted with asterisks, we used a random linear code, i.e., $z$ binary parity check constraints drawn in an equiprobable manner.

TABLE I: Number of deletions and code parameters for each setup.

\begin{tabular}{cccccccc}
\hline & $k$ & $n$ & $l_{1}$ & $l_{2}$ & $n_{c}$ & $z$ & $R$ \\
\hline Setup 1 & 3 & 60 & 5 & 3 & 4 & 4 & 0.650 \\
Setup 2 & 3 & 60 & 5 & 3 & 4 & 8 & 0.717 \\
Setup 3 & 3 & 60 & 5 & 3 & 4 & 12 & 0.783 \\
Setup 4 & 4 & 60 & 5 & 3 & 4 & 16 & 0.850 \\
Setup 5 & 7 & 378 & 9 & 7 & 6 & 42 & 0.365 \\
Setup 6 & 7 & 486 & 9 & 9 & 6 & $50^{*}$ & 0.325 \\
Setup 7 & 10 & 2800 & 20 & 20 & 7 & $60^{*}$ & 0.135 \\
\hline
\end{tabular}

TABLE II: List size after each step.

\begin{tabular}{ccccccc}
\hline & $\bar{r}_{1}$ & $\bar{r}_{3}$ & $\bar{r}_{4}$ & $\bar{r}_{6}$ & $\max r_{6}$ & $r_{6}>1$ \\
\hline Setup 1 & 1.87 & 1.92 & 1.42 & 1.003 & 3 & 3256 \\
Setup 2 & 1.87 & 1.92 & 1.42 & 1.000 & 2 & 25 \\
Setup 3 & 1.87 & 1.92 & 1.42 & 1 & 1 & 0 \\
Setup 4 & 3.39 & 6.18 & 2.53 & 1 & 1 & 0 \\
Setup 5 & 11.51 & 74.43 & 3.42 & 1 & 1 & 0 \\
Setup 6 & 11.20 & 28.64 & 2.55 & 1 & 1 & 0 \\
Setup 7 & 12.76 & 26.16 & 1.57 & 1 & 1 & 0 \\
\hline
\end{tabular}

Table II shows the list sizes of the number of candidates at the end of various steps of the decoding process. Recall that $r_{1}$ is the number of candidate block-deletion patterns at the end of step $1, r_{3}$ is the number of pairs $(\hat{Y}, \boldsymbol{A})$ at the end of step $3, r_{4}$ is the number of pairs $(\tilde{Y}, \tilde{\boldsymbol{A}})$ at the end of step 4 , and $r_{6}$ is the number of sequences $\hat{X}$ in the final list. Over $10^{6}$ trials, the average of $r_{i}$ is denoted by $\bar{r}_{i}$, the column $\max r_{6}$ shows the maximum size of the final list, and the column $r_{6}>1$ shows the number of trials for which $r_{6}>1$.

The first three setups have identical parameters, except for the number of Reed-Solomon parity checks. We observe that adding more parity check constraints increases the rate and improves decoding performance by reducing the number of trials with list size greater than one.
The fourth setup is precisely the code described in Example 1. It has the same values of $\left(n_{c}, l_{1}, l_{2}\right)$ as the first three setups but with a larger number of deletions and parity check constraints. We observe that increasing the number of deletions (with $n_{c}, l_{1}, l_{2}$ unchanged) increases the average number of candidates in the different decoding steps. In general, choosing $l_{1} \geq k$ ensures that the average list size after step 1 is small.

The fifth setup is a more practical code with length $n=$ 378 , and $k=7$ deletions. Though the final list size is always one, there are a large number of candidates at the end of the third step; this increases the decoding complexity. Comparing this with setup six, we observe that increasing $l_{2}$ significantly reduces the number of candidates at the end of the third step. This is because of the increase in the number of chunk-string VT constraints, which allows the decoder to eliminate more candidates while determining chunk boundaries.

The last setup is a relatively long code. Although the average number of candidates in each of the decoding steps is not very high, a small fraction of trials have a very large number of candidates, resulting in considerably slower decoding for these trials. For future work, an interesting direction is to consider lower-complexity decoders that allow for an early elimination of highly unlikely candidates. This would limit the number of candidates at the end of each decoding step at the expense of introducing a probability of error, i.e., a non-zero probability that the final list does not contain the true $X$ sequence.

\section{ACKNOWLEDGMENT}

We thank Jossy Sayir for kindly making his Reed-Solomon code simulator available to us.

\section{REFERENCES}

[1] G. Cormode, M. Paterson, S. C. Sahinalp, and U. Vishkin, "Communication complexity of document exchange," in Proc. ACM-SIAM Symp. on Discrete Algorithms, pp. 197-206, 2000.

[2] S. M. S. Tabatabaei Yazdi and L. Dolecek, "Synchronization from deletions through interactive communication," IEEE Trans. Inf. Theory, vol. 60, pp. 397-409, Jan. 2014.

[3] R. Venkataramanan, V. N. Swamy, and K. Ramchandran, "Lowcomplexity interactive algorithms for synchronization from deletions, insertions, and substitutions," IEEE Trans Inf. Theory, vol. 61, no. 10, pp. 5670-5689, 2015.

[4] N. Ma, K. Ramchandran, and D. Tse, "Efficient file synchronization: A distributed source coding approach," in Proc. IEEE Int. Symp. Inf. Theory, 2011.

[5] R. R. Varshamov and G. M. Tenengolts, "Codes which correct single asymmetric errors," Automatica i Telemekhanica, vol. 26, no. 2, pp. 288 292, 1965.

[6] V. I. Levenshtein, "Binary codes capable of correcting deletions, insertions and reversals," Doklady Akademii Nauk SSSR, vol. 163, no. 4, pp. 845-848, 1965.

[7] A. Orlitsky, "Interactive communication of balanced distributions and of correlated files," SIAM J. Discrete Math., vol. 6, no. 4, pp. 548-564, 1993.

[8] A. Orlitsky and K. Viswanathan, "One-way communication and errorcorrecting codes," IEEE Trans. Inf. Theory, vol. 49, no. 7, pp. 1781$1788,2003$.

[9] N. J. A. Sloane, "On single-deletion-correcting codes," in Codes and Designs, Ohio State University (Ray-Chaudhuri Festschrift), pp. 273291, 2000. Online: https://arxiv.org/abs/math/0207197.

[10] M. Abroshan, R. Venkataramanan, and A. Guillén i Fàbregas, "Multilayer codes for synchronization from deletions," 2017. (Online) https://arxiv.org/abs/1705.06670. 